\title{
Use of radionuclide scanning to estimate size of spleen in vivo
}

\author{
B ZHANG, S M LEWIS \\ From the Department of Haematology, Royal Postgraduate Medical School, London
}

SUMMARY Splenic ultrasound was performed and spleen scans obtained by a rectilinear scanner after injection of In-113m-labelled heat damaged red cells on 14 patients with various haematological disorders. Correlation was assessed between the measured volume of the operatively removed spleens, their volumes as calculated from the ultrasound data, and their preoperative dimensions of length, circumference, and area on the scans. The relation of scan to volume was best defined by an equation $\mathrm{V}=9.88 \mathrm{~A}-534$, where $\mathrm{V}=$ volume of spleen $\left(\mathrm{cm}^{3}\right)$ and $\mathrm{A}=\operatorname{area}\left(\mathrm{cm}^{2}\right)$ on posterior scan. The mean percentage difference between calculated and actual spleen volume was 0.2 (SD 6.7)\%. Average spleen density was established as $1.04 \mathrm{~g} / \mathrm{cm}^{3}$. The formula was shown to be reliable in the range of splenic area $c 73-450 \mathrm{~cm}^{2}$ - that is, volume $c 185-4000 \mathrm{~cm}^{3}$. This range includes the average normal sized spleens and the splenomegalies that are most often found in clinical practice. The formula is not valid when the spleen is smaller than normal. As the method depends on measurement of the spleen image shown by scintigraphs, it cannot be used when there is functional asplenia.

The estimation of spleen size in vivo is often important in the diagnosis, treatment, and prognosis of a variety of disorders. Measurement of spleen size by means of physical examination of the abdomen is unreliable as minor degrees of splenic enlargement are often indetectable by palpation, and, in some instances, even a grossly enlarged spleen might be missed. ${ }^{1}$

For this reason, scintigraphic methods were developed for spleen imaging. Initially these studies provided only limited measures of splenic length or area, or both, from the splenic image on the scan. ${ }^{2}$ It was only from the mid 1960 s that methods for the estimation of the spleen volume were developed. These were based on measurement of one or more dimensions, such as length, height, width, circumference or area of the spleen image on the scan, using artifically damaged red blood cells or colloid. ${ }^{3-7}$ None of these methods, however, has been fully evaluated or generally accepted.

Well defined splenic images are obtained by means of a rectilinear scanner after administration of radionuclide labelled heat damaged red cells. ${ }^{8}$ This was used in the present study to develop a method for

Accepted for publication 3 December 1986 estimation of spleen volume from scintigraphy, using direct measurement of the removed spleen as a primary reference. Ultrasonography also provides a method for estimating spleen volume. ${ }^{9}$ If these two non-invasive methods are shown to be equally valid and comparable it should then be possible to use either as a reference or calibrator for determining the validity of calculating spleen volumes from images obtained by other scintigraphic procedures.

\section{Patients and methods}

Fourteen patients were referred to the department of haematology at this hospital for radionuclide investigations of their splenic function before they underwent splenectomy for various haematological disorders. None of the patients was subjected to additional procedures. There were nine women and five men, aged between 15 and 63 years (median age 50 years). The conditions included three cases of myelofibrosis, three cases of immune thrombocytopenia, two cases each of chronic granulocytic leukaemia and portal hypertension, hereditary spherocytosis, leishmaniasis and idiopathic splenomegaly.

In most cases splenectomy was performed shortly after the radionuclide study - that is, from three days to three weeks. In two patients (one with hairy cell 08 
leukaemia and the other with myelofibrosis) the spleen scans were done at about three months before splenectomy; in both, their clinical condition remained stable and they received no chemotherapy or radiotherapy during this period, and there was no evidence of any change in the size of their spleens by palpation.

Autologous red cells collected into acid-citratedextrose anticoagulant solution were labelled with In-113m by the method of Danpure and Osman ${ }^{10}$ and heat damaged by the method of Marsh et al. ${ }^{11}$

Spleen scan was performed 20 minutes after the intravenous administration of $18.5 \mathrm{MBq}(500 \mathrm{uCi})$ of the In-113m labelled heat damaged red cells using a dual detector scanner (Dual Magnascanner) with four inch $(10 \mathrm{~cm})$ focusing collimators, specially designed for use with high energy emitters. A $0.2 \mathrm{~cm}$ line spacing was used. Every effort was made to select the high energy portion of the In- $113 \mathrm{~m}$ photo peak to minimise background scatter. In each case the photoscans or paper scans, or both, were taken in the anterior, posterior, and lateral positions.

The photo or paper scans were illuminated on a viewing box; and the outline of the spleen on the scan was determined and transcribed on tracing paper. The line was smoothed, when necessary, to eliminate any minor irregularity of the scan edge. Three measurements were made on each projection-that is, maximal length, area (measured by planimeter), and circumference of the spleen image, as well as on the anterior overlapping posterior projection obtained by using both detectors for the anterior and posterior spleen scans simultaneously.

During operations, the splenic artery and vein were carefully clamped simultaneously. After their removal the spleens were weighed immediately and then their volumes were measured by water displacement.

The density of each removed spleen was calculated. The maximum length of the spleen measured from the posterior scan, as well as the anterior plus posterior (anteroposterior) scan, and the actual length measured on the removed spleen were compared in each case. The various parameters measured on each projection were plotted as a function of the volume of the removed spleens. Using a Hewlett-Packard 9810A computer, the linear, power, exponential, parabolic and cubic regression equations and their individual correlation coefficients were determined.

Before operation the patients also underwent ultrasonography on the same day, or within two days of the scintigraphy. The apparatus used for this was a Rohnar 5580 diagnostic ultrasound system with a 3.5 $\mathrm{MHz} 10 \mathrm{~cm}$ focused transducer $(2 \mathrm{~cm}$ in diameter). The longest splenic longitudinal axis was determined; scans were obtained at right angles to this axis

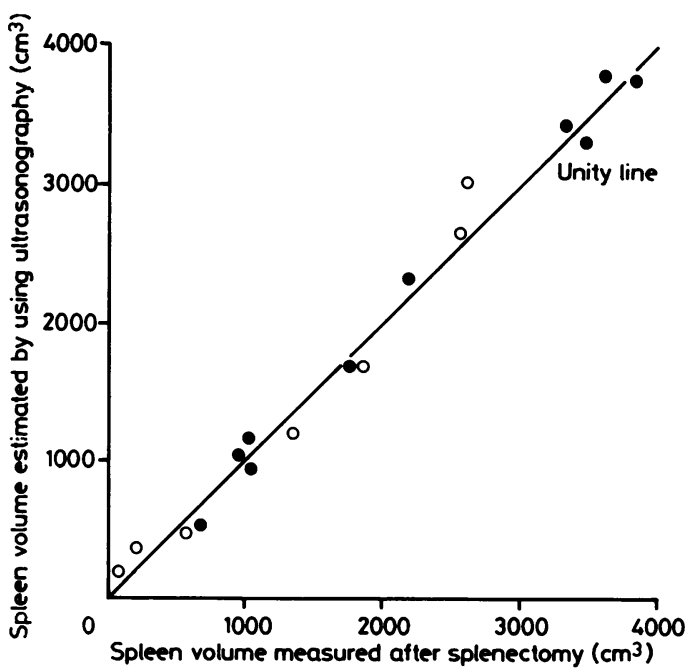

Fig 1 Relation between measurements of spleen volume derived from ultrasound and after splenectomy in 10 patients in this series ( ) and in seven patients studied by Kardel et $a^{9}(O) ; r=0.9976, p<0.001$.

throughcut the entire length at constant intervals of $2 \mathrm{~cm}$ and the volume of the spleen was calculated from the measurements of these transverse cross sections according to the method and formula of Kardel et $_{\text {al. }}{ }^{9}$

\section{Results}

Mean difference between spleen volume calculated from ultrasound and measured directly after splenectomy was $-1(5 \cdot 1) \%$, with individual differences

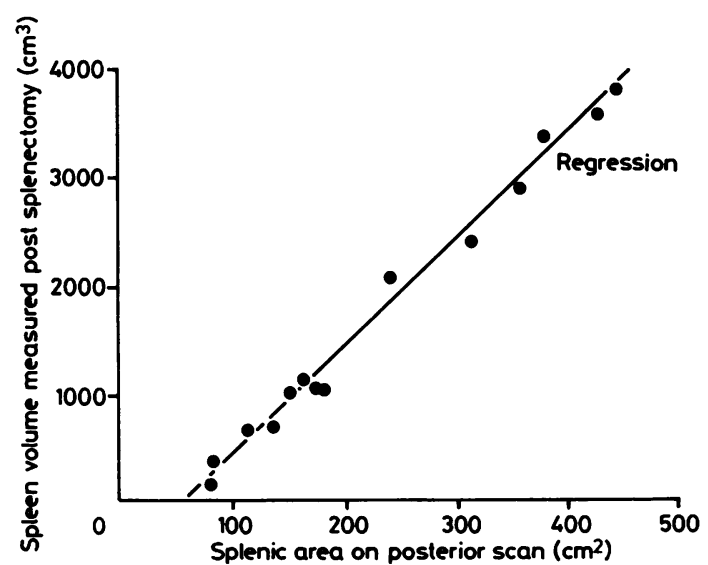

Fig 2 Relation between splenic area on posterior spleen scan and spleen volume measured after splenectomy in 14 patients studied. Continuous line represents regression line, $V=9.88 A-534(r=0.9961, p<0.001)$. 


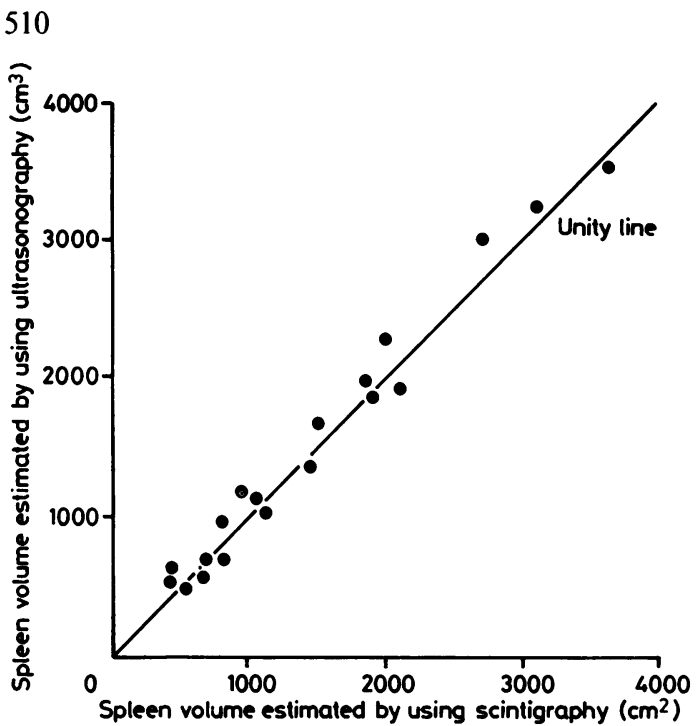

Fig 3 Relation between spleen volumes estimated by ultrasound and from scintigraphic scan on 14 patients in this series and five additional patients; $r=0.9948, p<0.001$.

ranged between +5.9 and $-9.1 \%$ (fig 1 ). The Wilcoxon's rank sum test showed that there were no significant differences; $p>0.05$.

Five different regression equations (linear, power, exponential, parabolic and cubic) were used to calculate spleen volumes from the scintigraphic images using measurements of posterior length; posterior and lateral circumference; and area measured on anterior, posterior, anteroposterior and lateral scans. These were compared with the volumes measured directly after splenectomy. Correlation coefficients ranged from 0.86 to 0.99 , the best correlation being provided by area from posterior and anteroposterior scans. Fig 2 shows the relation between area from posterior scans and volumes measured directly after splenectomy. There was excellent correlation; $r=$ $0.9961 \mathrm{p}<0.001$. The best correlation with calculated spleen volume was obtained by linear, parabolic, and cubic regression equations. From the computed data (fig 2) the linear equation based on the area of the spleen obtained from the posterior scan was chosen for the estimation of the spleen volume. This equation is $\mathrm{V}=9.88 \mathrm{~A}-534$, where $\mathrm{V}=$ volume of spleen $\left(\mathrm{cm}^{3}\right)$ and $\mathrm{A}=$ area of spleen on posterior scan $\left(\mathrm{cm}^{2}\right)$. The spleen areas were 73 to $442 \mathrm{~cm}^{2}$. At operation their volumes were $186-3585 \mathrm{ml}$ (mean $1716 \mathrm{ml}$ ); percentage differences between volume derived from the formula and by direct measurement ranged between -11.3 and $+13.8 \%$ (mean $0.2 \%$, SD 6.7 ); the modal difference was $5 \cdot 1 \%$. Fig 3 shows comparison of calculated volumes with volumes derived from ultrasound.
Zhang, Lewis $\stackrel{\complement}{\frac{\Omega}{\bar{\Xi}}}$

The comparison of the length of the spleens measured on the posterior scans and the length of the spleens measured after splenectomy showed an extremely good coincidence with percentage differences between them of -6.3 to +6.9 (mean $\overline{0}$ $-0 \cdot 1 \%$, SD $3 \cdot 1 \%$ ). Wilcoxon's rank sum test showed $\frac{c}{0}$ no significant difference between these two sets of lengths (total of ranks $=41 \cdot 5, p>0.05$ ). From the $\underset{Q}{\mathbb{Q}}$ weight:volume ratio, the density of the spleen was calculated. The mean density was 1.04 (SD 0.016) $\mathrm{g} / \mathrm{cm}^{3}$. क

\section{Dis:ussion}

An equation was developed for calculating spleen volume from scintigraphic scans based on measurements from 14 patients who had undergone splenectomy of and who had had their spleens scanned shortly or beforehand. The patients had various haematological $\varnothing$ disorders. The sizes of the spleens varied from normal 음 to grossly enlarged. In this study the spleei:s of the patients were imaged in posterior, anterior, ante- $\bar{z}$ roposterior and lateral views. The three sets of measurements of length, circumference, and area of the $\overrightarrow{0}$ spleen images obtained from the different scans men- $\infty$ tioned above were correlated with the size of the spleens measured postoperatively, and five different equations were applied to each pair. Spleen area obtained in the posterior and anteroposterior views showed the best correlation with spleen size by linear, parabolic, and cubic regression equations.

The linear equation was favoured as being most $\overrightarrow{\hat{O}}$ convenient in routine practice. The posterior scan has $\exists$ the advantage that the possible variation in image size which occurs with varying spleen detector distance can be minimised, as in this position the distance is relatively constant from patient to patient. ${ }^{12}$ The excellent correlation between the maximal lengths of 3 the spleen images on the scans and those measured from the removed spleens has established that the boundary of the spleen image on the scan represented the true spleen area in posterior view. Furthermore, it is hoped that the equation might also be used in con- $\frac{7}{0}$ junction with any scintillation camera which cannot image the spleen from both anterior and posterior $N$ views .imultaneously.

Accordingly, the linear equation $\mathrm{V}=9 \cdot 88 \mathrm{~A}-534$ N $\left(\mathrm{cm}^{3}\right)$ based on the spleen area on the posterior spleen scan became the best choice for the estimation of the spleen volume in vivo. The comparison with the spleen volume derived from ultrasonography and the spleen volume measured at operation showed that ${ }^{-}$ this method was precise and reproducible in the range which was included. It must be remembered, how- $\overrightarrow{\mathbb{D}}$ ever, that extrapolating a regression line at either end $\stackrel{?}{\mathbb{\Phi}}$ may not be reliable beyond the measured data. On the $\triangle$ basis of the data obtained from this study, therefore, 
the equation can only be used reliably for estimation of spleen size when the spleen area in posterior scan is within a range of about $73-450 \mathrm{~cm}^{2}$. An area of $73 \mathrm{~cm}^{2}$ represents a volume of $187 \mathrm{~cm}^{2}$, which is well within the normal range. The normal range of the area of the posterior spleen image has been reported previously as being $35-85 \mathrm{~cm}^{2},{ }^{2}<80 \mathrm{~cm}^{2},{ }^{3} 24-82$ $\mathrm{cm}^{2},{ }^{13}$ and $47-79 \mathrm{~cm}^{3} .{ }^{14}$ From the equation, a maximum normal splenic volume of about $80 \mathrm{~cm}^{2}$ is calculated to be $256 \mathrm{~cm}^{3}$. Thus our method is valid for calculating the spleen volume accurately when the spleen is only marginally enlarged; the formula is not valid, when the spleen has an area below about 73 $\mathrm{cm}^{2}$. The validity for measurements beyond the upper limit of $4000 \mathrm{~cm}^{3}$ remains to be determined, but fortunately, the range in which the formula is reliable covers the spleen volumes which are most often met with in splenomegaly in clinical practice.

Several factors indicate that this formula may be more reliable in clinical application than previously proposed equations suggest. Firstly, it is based on a study of patients who have undergone splenectomy, whereas the equations established by Fischer and Wolf, ${ }^{3}$ Spencer, ${ }^{4}$ Mattsson, ${ }^{6}$ and Samuels ${ }^{15}$ were based on theoretical models alone. Secondly, the change in the spleen volume was not significant in the short time between the spleen scan and splenectomy, whereas only spleens obtained at necrospy were used for establishing the equations proposed by Rollo and DeLand. ${ }^{5}$ Thirdly, at operation the splenic vein and artery were clamped simultaneously so that there was no noticeable loss of blood, whereas there was no discription of this in the reports by Rollo and DeLand, ${ }^{5}$ Ingeberg et al, ${ }^{7}$ and Larson et al. ${ }^{13}$

Values for gravity density of the spleen have differed widely in various studies. It has been reported as $1 \cdot 00,{ }^{5} 1 \cdot 04,9^{16} 1 \cdot 06,{ }^{317}$ and $1 \cdot 14 .^{18}$ In our study we found that the average density of the spleen from 14 patients was $1.04 \mathrm{~g} / \mathrm{cm}^{3}$ (SD $0.016 \mathrm{~g} / \mathrm{cm}^{3}$ ). It is therefore possible to convert splenic volume to weight by a factor of 1.04.

It is appreciated that rectilinear scanning is not widely available. The demonstration of reliability of measurement of spleen volume by ultrasonography and the comparability of results from the scan when an appropriate formula is applied, however, indicates that ul:rasound scan can be used as a reference for establishing the validity of the formula, or a modification thereof, when an alternative imaging technique or another radionuclide are used.

In applying the equation, it should be borne in mind that it is the ability of the spleen to sequestrate and phagocytose deliberately damaged red cells from the circulation which permits the visualisation of the spleen by scanning. Therefore, if there is a functional asplenia, as has been ciescribed by Spencer et al, ${ }^{19}$ this equation will not be valid; non-visualisation of part or the whole of a spleen makes it impossible to measure the spleen area correctly; but this limitation would apply equally to all methods based on imaging.

We thank Professor L Blumgart and Mr J Lynn of the department of surgery, Royal Postgraduate Medical School, for their cooperation during the performance of splenectomies, and Dr I A Lampert and Professor N Wright of the department of histopathology for the facilities provided for the measurement of the removed spleens. We are grateful to $\mathrm{Mr} \mathbf{M}$ Deenmamode, Mr G Hooker, Miss M G William, and Miss A Henry for their technical help with the radionuclide procedures and to Dr $\mathrm{S}$ Leeman for help with ultrasonography.

\section{References}

1 Westin J, Lanner LO, Larsson A, Weinfeld A. Spleen size in polycythaemia. Acta Med Scand 1972;191:263-71.

2 Holzbach RT, Clark RE, Shipley RA, Kent WB, Lindsay GE. Evaluation of spleen size by radioactive scanning. $J$ Lab Clin Med 1962;60:902-13.

3 Fischer J, Wolf R. Quantitative estimation of spleen size by scintigraphy. German Medical Monthly 1964;9:62-8.

4 Spencer RP. Relationship of surface area on roentgenograms and radioisotopic scans to organ volumes. J Nucl Med 1967; 8:785-91.

5 Rollo FD, DeLand FH. The determination of spleen mass from radionuclide images. Radiology 1970;97:583-7.

6 Mattsson O. Scintigraph spleen volume calculation. Acta Radiol (Diagn) (Stockh) 1982;23:471-7.

7 Ingeberg S, Stockel M, Sorensen PJ. Prediction of spleen size by routine radioisotope scintigraphy. Acta Haematol 1983;69: 243-8.

8 Bowring CS. Quantitative radioisotope scanning and its use in haematology. Clin Haematol 1977;6:625-37.

9 Kardel T, Holm HH, Rasmussen N, Mortensen T. Ultrasonic determination of liver and spleen volumes. Scand J Clin Lab Invest 1971;27:123-8.

10 Danpure HJ, Osman S. Cell labelling and cell damage with indium-111; acetyl acetone-an alternative to indium-111 oxine. Br J Radiol 1981;54:597-601.

11 Marsh GW, Lewis SM, Szur L. The use of $\mathrm{Cr}-51$ labelled heat damaged red cells to study splenic function. 1. Evaluation of Method. Br J Haematol 1966;12:161-6.

12 Sigel RM, Becker DV, Hurley JR. Evaluation of spleen size during routine liver imaging with Tc-99m and the scintillation camera. J Nucl Med 1970;11:689-92.

13 Larson SM, Tuell SH, Moores KD, Nelp WB. Dimensions of the normal spleen scan and prediction of spleen weight. $J$ Nucl Med 1971;12:123-6.

14 Hegde UM, Wiliams ED, Lewis SM, Szur L, Glass HI, Pettit JE. Measurement of splenic red cell volume and visualization of the spleen with Tc-99m. J Nucl Med 1973;14:769-71.

15 Samuels LD. Estimation of spleen weight from posterior radioisotope scans. J Assoc Can Radiol 1969;20:192-3.

16 Rasmussen SN, Christensen BE, Holm HH, Kardel T, Strigsby B, Larsen M. Spleen volume determination by ultrasonic scanning. Scand J Haematol 1973;10:298-304.

17 Levesque MJ, Groom AC. Fast transit of red cells and plasma in contracted versus relaxed spleens. Can J Physiol Pharmacol 1981;59:53-8.

18 Aito $\mathrm{H}$. The estimation of the size of the spleen by radiological methods. Ann Clin Res 1974;15:1-54

19 Spencer RP, Dhawan V, Suresh K, Antar MA, Sziklas JJ, Wasserman I. Causes and temporal sequence of onset of functional asplenia in adults. Clin Nucl Med 1978;3:17-8.

Requests for reprints to: Dr S M Lewis, Department of Haematology, Royal Postgraduate Medical School, Du Cane Road, London W12 0HS, England. 